\title{
FATORES OBSTETRICOS ASSOCIADOS AO BAIXO PESO AO NASCER
}

\author{
Umberto Gazi Lippi* \\ Antonio Suzart de Andrade" \\ José Ricardo D. Bertagnon * \\ Evaldo Melo*
}

LIPPI, U.G. et. al. Fatores obstétricos associados ao baixo peso ao nascer. Rev. Sá́de públ, S. Paulo, 23: 382-7, 1989.

RESUMO: Estudou-se um contingente de 19.446 eventos obstétricos consecutivos, dos quais foi possível obter dados analistuveis em 18.804. O baixo peso ao nascer ocorreu em 15,93\% dos recém-natos. Foi possível encontrar associaçoes estatisticamente significativas com os elementos estudados, ou seja, com: idade materna, assisténcia pré-natal, número de gestaçóes prévias, hábito de fumar e idade gestacional ao parto. Propöe-se medidas para atenuar o problema, quais sejam: programas educacionais sobre reproduçăo humana para adolescentes, programas destinados a divulgar a nocividade do hábito de fumar, extensăo da assisténcia prét-natal, programas médicos do bloqueio do parto prematuro, tudo associado a outras, de caráter sócio-economico.

DESCRITORES: Baixo peso ao nascer. Prevalencia. Fatores de risco.

\section{INTRODUÇÃO}

O baixo peso ao nascer (BPN-menos de $2.500 \mathrm{~g}$ ), quer em nascidos de pré-termo, adequados para a idade gestacional, quer naqueles desnutridos intra-uterinamente, representa um grave problema de saúde pública. Associa-se fortemente ao obituário neonatal e a transtornos do desenvolvimento neuropsíquico de muitas crianças. Schwarcz e col. ${ }^{10}$ fizeram estudo colaborativo em 59 maternidades de 11 países latino-americanos e mostraram um total de $9,2 \%$ de recém-natos de baixo peso, sendo 1,2\% de muito baixo peso (menos de $1.500 \mathrm{~g}$ ). Esses mesmos autores mostraram que esses $9,2 \%$ de recém-nascidos contribuíram com 78\% da mortalidade neonatal precoce (até 7 dias de vida). No Chile, Mardones ${ }^{7}$ revelou ser de $11 \%$ a freqüência de BPN. Yoshida-Ando e Mendoza-Pérez ${ }^{11}$ apontaram taxas de $16 \%$ de BPN em um grande hospital do México, entre 17.000 nascidos vivos. Já Hutchins e col. ${ }^{3}$ mostraram que nos Estados Unidos a taxa foi de 6,9\%, em 1980. Schuler e Klinger ${ }^{9}$ afirmaram que em 1981, na Hungria, ocorreram $10,2 \%$ de nascimentos com baixo peso. E evidente a falta de homogeneidade entre os números apresentados, com desvantagem para os países menos desenvolvidos. Aliás, Schwarcz e $\mathrm{col}^{10}$ citam que em 1979 nasceram no mundo 21 milhöes de crianças com baixo peso, das quais $90 \%$ em países subdesenvolvidos.

A importante dimensāo do problema levou muitos estudiosos a pesquisarem fatores associados ao evento. Assim, Mardones ${ }^{7}$ e Barros e col. ${ }^{1}$ encontraram maior freqüência de BPN entre mães adolescentes. Schuler e Klinger ${ }^{9}$ bem como Berenzin e col. ${ }^{2}$ registraram a influência negativa da assistência pré-natal ausente ou precária em associação com o evento. Miller ${ }^{8}$, Barros e col. ${ }^{1}$ e Schuler e Klinger ${ }^{9}$ associaram a ocorrência do BPN ao hábito de fumar. Já analisando a paridade das mães de recém-natos com menos de $2.500 \mathrm{~g}$, Miller ${ }^{8}$, Berezin e col. ${ }^{2}$ não encontraram qualquer associaçāó significativa da mesma com o BPN. A idade gestacional está reconhecidamente associada ao evento. Tanto assim que Mardones ${ }^{7}$ notou que a grande maioria dessas crianças nascia com idade inferior a 37 semanas.

O objetivo do presente estudo é avaliar a importância de alguns dos fatores obstétricos já mencionados, quando cotejados ao baixo peso ao nascer.

\section{MATERIAL E MÉTODO}

Estudou-se no período de 1/11/1980 a $31 / 8 / 198519.446$ pacientes, correspondente ao to- 
tal da população atendida na instituição. Os dados foram coletados pela História Clínica Perinatal, conforme descrita por Lippi e col. ${ }^{4}$, e armazenados em um computador IBM de grande porte.

Procurou-se avaliar a relação entre a ocorrência de BPN e os seguintes fatores: idade materna, acompanhamento pré-natal, inclusive número de consultas, número de gestações prévias, hábito de fumar e numero de cigarros consumidos por dia e idade gestacional ao parto."

Para análise estatística efetuou-se o teste de contingência. Para qualquer decisão foi exigido $P$ inferior a $5 \%$.

\section{RESULTADOS}

Freqũência de recém-nascidos com baixo peso ao nascer

Foram recuperados os dados relacionados ao peso ao nascer em 18.804 partos. Desse total, 2.996 recém-nascidos pesaram menos de $2.500 \mathrm{~g} \mathrm{o}$ que significa uma freqüĉncia de $15,9 \%$.

Relaçăo entre idade materna e freqüência de recém-nascidos de baixo peso

A Tabela 1 mostra que a maior freqüência de BPN $(19,1 \%)$ verificou-se entre as adolescentes muito jovens (abaixo dos 17 anos) e entre aquelas entre 17 e 19 anos completos (16,8\%). Todas as adolescentes foram responsáveis por
$17,4 \%$ de recém-natos de baixo peso, enquanto as mães com 20 anos e mais responsabilizaram-se por $15,6 \%$ dessas crianças. A aplicação do teste estatístico mostrou significância (chi-quadrado $=10,60$ para alfa $1,0 \%$ e $2 \mathrm{gl}$.) denotando associação entre idade materna e BPN. Da mesma forma, significativo foi o resultado (chi-quadrado $=7,31$ para alfa $1,0 \%$ e $1 \mathrm{gl}$.) quando se comparou o grupo de adolescentes muito jovens e todas as outras mães.

\section{Relação entre acompanhamento pré-natal e fre- qũência de recém-nascidos de baixo peso}

A Tabela 2 mostra que as mães sem assistência pré-natal tiveram 17,1\% de crianças com BPN enquanto que as assistidas apresentaram freqüên. cia de 14,0\%. O teste estatístico aplicado demonstrou alta significância (chi-quadrado $=31,20$ para alfa $0,5 \%$ e $1 \mathrm{gl}$.).

\section{TABELA 2}

Relação entre acompanhamento pré-natal e frequiência de recém-nascidos de baixo peso

\begin{tabular}{lccccc}
\hline & \multicolumn{3}{c}{ Acompanhamento pré-natal } \\
\cline { 2 - 5 } Peso (g) & \multicolumn{2}{c}{ Sim } & & \multicolumn{2}{c}{ Não } \\
\cline { 2 - 5 } \cline { 5 - 6 } & No & $\%$ & & No & $\%$ \\
\hline$<2.500$ & 949 & 14,0 & 1.952 & 17,1 \\
$\geqslant 2.500$ & 5.838 & 86,0 & 9.451 & 83,0 \\
\hline Total & 6.787 & 100,0 & 11.403 & 100,0 \\
\hline
\end{tabular}

TABELA 1

Relação entre idade materna e frequiência de recém-nascidos de baixo peso

\begin{tabular}{|c|c|c|c|c|c|c|}
\hline \multirow{3}{*}{ Peso (g) } & \multicolumn{6}{|c|}{ Idade materna (anos) } \\
\hline & \multicolumn{2}{|c|}{ até 16} & \multicolumn{2}{|c|}{$17-19$} & \multicolumn{2}{|c|}{20 e mais } \\
\hline & No & $\%$ & No & $\%$ & No & $\%$ \\
\hline$<2.500$ & 179 & 19,1 & 465 & 16,8 & 2.352 & 15,6 \\
\hline$\geqslant 2.500$ & 759 & 80,9 & 2.298 & 93,2 & 12.751 & 84,4 \\
\hline Total & 938 & 100,0 & 2.763 & 100,0 & 15.103 & 100,0 \\
\hline
\end{tabular}


TABELA 3

Relação entre total de controles pré-natal e frequiência de recém-nascidos de baixo peso

\begin{tabular}{|c|c|c|c|c|c|c|c|c|}
\hline \multirow{3}{*}{ Peso (g) } & \multicolumn{8}{|c|}{ Número de controles de pré-natal } \\
\hline & \multicolumn{2}{|c|}{$0-2$} & \multicolumn{2}{|c|}{$3-4$} & \multicolumn{2}{|c|}{$5-6$} & \multicolumn{2}{|c|}{7 e mais } \\
\hline & Nọ & $\%$ & Nọ & $\%$ & Nọ & $\%$ & N! & $\%$ \\
\hline$<2.500$ & 2.187 & 17,8 & 322 & 19,1 & 230 & 10,4 & 170 & 8,2 \\
\hline$\geqslant 2.500$ & 10.087 & 82,2 & 1.360 & 80,9 & 1.977 & 79,6 & 1.914 & 91,8 \\
\hline Total & 12.274 & 100,0 & 1.682 & 100,0 & 2.207 & 100,0 & 2.084 & 100,0 \\
\hline
\end{tabular}

Relação entre o número de consultas de pré-natal e a frequêencia de recém-nascidos de baixo peso

A Tabela 3 mostra que o número de consultas pré-natal, a partir de cinco, faz cair drasticamente a freqüência de recém-nascidos de baixo peso. Assim entre 0 e 2 consultas esse valor é de $17,8 \%$, para 3 e 4 consultas é de $19,1 \%$ para 5 e 6 ć dy $10,4 \%$ e para 7 consultas e mais é de $8,2 \%$. A aplicaçẩo do teste estatístico revelou alta significância (chi-quadrado $=189,54$ para alfa $0,5 \%$ com $3 \mathrm{gl}$.) denotando a associação entre o número de consultas e o BPN.

Relação entre o número de gestaçōes prévias e a freqüência de recém-nascidos de baixo peso

Na Tabela 4 pode-se ver a freqüência de BPN com vistas ao número de gestaçōes prévias das mães. Os percentuais ali expostos são próximos, não deixando muito clara a associação, mas a análise estatística revclou significância (chi-quadrado $=11,62$ para alfa $1 \%$ e $3 \mathrm{gl}$.)

Relação entre o hábito de fumar e a frequêencia de recém-nascidos com baixo peso ao nascer

A Tabela 5 mostra que a freqüência de BPN é maior $(19,8 \%)$ entre mães fumantes que entre as nāo-fumantes $(12,3 \%)$. A significância estatística foi elcvada (chi-quadrado $=128,58$ para alfa $0,5 \%$ e $1 \mathrm{gl}$.).

\section{TABELA 5}

Relação entre fumo e frequiência de recém-nascido de baixo peso

\begin{tabular}{lccccc}
\hline \multirow{2}{*}{ Peso $(\mathrm{g})$} & \multicolumn{2}{c}{ Fumante } & & \multicolumn{2}{c}{ Não fumante } \\
\cline { 2 - 3 } & No & $\%$ & & No & $\%$ \\
\hline$<2.500$ & 1.017 & 19,8 & & 883 & 12,3 \\
$\geqslant 2.500$ & 4.124 & 80,2 & 6.296 & 87,7 \\
\hline Total & 5.141 & 100,0 & & 7.179 & 100,0 \\
\hline
\end{tabular}

TABELA 4

Relação entre número de gestações prévias e frequiência de recém-nascidos de baixo peso

\begin{tabular}{|c|c|c|c|c|c|c|c|c|}
\hline \multirow{3}{*}{ Peso (g) } & \multicolumn{8}{|c|}{ Número de gestaçōes prévias } \\
\hline & \multicolumn{2}{|c|}{0} & \multicolumn{2}{|c|}{$1-3$} & \multicolumn{2}{|c|}{4 e 5} & \multicolumn{2}{|c|}{6 e mais } \\
\hline & No & $\%$ & Nọ & $\%$ & No & $\%$ & No & $\%$ \\
\hline$<2.500$ & 779 & 16,3 & 1.383 & 14,9 & 417 & 17,6 & 353 & 16,1 \\
\hline$\geqslant 2.500$ & 3.999 & 83,7 & 7.865 & 85,1 & 1.957 & 82,4 & 1.836 & 83,9 \\
\hline Total & 4.778 & 100,0 & 9.248 & 100,0 & 2.374 & 100,0 & 2.189 & 100,0 \\
\hline
\end{tabular}


TABELA 6

Relação entre número de cigarros diários e freqüência de recém-nascidos de baixo peso

\begin{tabular}{|c|c|c|c|c|c|c|c|c|c|c|}
\hline \multirow{3}{*}{ Peso $(\mathrm{g})$} & \multicolumn{10}{|c|}{ Número de cigarros diários } \\
\hline & \multicolumn{2}{|c|}{$1-5$} & \multicolumn{2}{|c|}{$6-10$} & \multicolumn{2}{|c|}{$11-15$} & \multicolumn{2}{|c|}{$16-20$} & \multicolumn{2}{|c|}{21 e mais } \\
\hline & N? & $\%$ & Nọ & $\%$ & No & $\%$ & Nọ & $\%$ & No & $\%$ \\
\hline$<2.500$ & 393 & 17,6 & 326 & 21,0 & 38 & 18,7 & 239 & 22,8 & 21 & 18,7 \\
\hline$\geqslant 2.500$ & 1.837 & 82,4 & 1.224 & 79,0 & 165 & 81,3 & 807 & 77,2 & 91 & 81,3 \\
\hline Total & 2.230 & 100,0 & 1.550 & 100,0 & 203 & 100,0 & 1.046 & 100,0 & 112 & 100,0 \\
\hline
\end{tabular}

Relação entre o número de cigarros diários e a freqūência de recém-nascidos de baixo peso

Na Tabela 6 pode-se observar que há uma tendência para crescer a freqüência do BPN com o aumento do consumo de cigarros. De fato essa associação fica evidente ao se aplicar o teste estatístico que revelou significância (chi-quadrado = 14,49 para alfa $1 \%$ e 4 gl.). Utilizando-se o mesmo teste, mas comparando a freqüência de BPN entre o grupo das não-fumantes e o das que fumam até 5 cigarros por dia, obteve-se chi-quadrado = 41,13 (para alfa $0,5 \%$ e $1 \mathrm{gl}$.), também significativo. De forma idêntica denotou-se significância estatística quando as comparaçōes foram feitas para o grupo de fumantes até 5 cigarros diários e o de consumidoras de 6 ou mais cigarros por dia (chi-quadrado $=11,85$ para alfa $0,5 \%$ e 1 gl.).

\section{Relação entre a idade gestacional ao parto e a freqũência de recém-nascidos de baixo peso}

A Tabela 7 mostra que a freqüência de baixo peso é de $46,3 \%$ quando a idade gestacional ao parto atinge até 36 semanas, caindo verticalmente para 8,2\% entre 37 e 40 semanas e mais ainda para $4,1 \%$ quando aquela atinge 41 semanas e mais. A significância estatística foi altíssima quando se aplicou o teste estatístico considerando os três grupos (chi-quadrado $=3.137,41$ para alfa $0,5 \% \mathrm{e}$ $2 \mathrm{gl}$.), bem como quando se considerou o grupo de até 36 semanas comparado ao de 37 e mais (chiquadrado $=3.114,12$ para alfa $0,5 \%$ e $1 \mathrm{gl}$.).

\section{COMENTÁRIOS}

Assinalou-se na parte introdutória deste trabalho a alta dimensão das taxas de recém-nascidos de baixo peso. A frequiência de $15,9 \%$ verificada

\section{TABELA 7}

Relação entre idade gestacional ao parto e frequiência de recém-nascidos de baixo peso

\begin{tabular}{|c|c|c|c|c|c|c|}
\hline \multirow{3}{*}{ peso (g) } & \multicolumn{6}{|c|}{ Idade gestacional ao parto (semanas) } \\
\hline & \multicolumn{2}{|c|}{ até 36} & \multicolumn{2}{|c|}{37 a 40} & \multicolumn{2}{|c|}{41 e mais } \\
\hline & No & $\%$ & Nọ & $\%$ & Nọ & $\%$ \\
\hline$<2.500$ & 1.607 & 46,3 & 907 & 8,2 & 87 & 4,1 \\
\hline$\geqslant 2.500$ & 1.861 & 53,7 & 10.079 & 91,8 & 2.037 & 95,9 \\
\hline Total & 3.468 & 100,0 & 10.986 & 100,0 & 2.124 & 100,0 \\
\hline
\end{tabular}


na população estudada revela-se semelhante àquelas de Yoshida-Ando e Mendoza-Pérez ${ }^{11}$ e de Schwarcz e col. ${ }^{10}$, relativos a populaçōes latino-americanas. $E$, n $\rho$ entanto, mais avantajada quando a comparação é feita com os dados de Miller $^{8}$ para populaçōes norte-americanas. Fica implícito que o esforço dos perinatólogos deve ser dirigido para o manuseio do problema. É de capital importância que haja uma conscientização de que não bastam medidas como o bloqueio do parto prematuro, o tratamento de desnutrição intra-uterina e os cuidados especiais com o recémnato. Há necessidade de medidas profiláticas que minimizem a ocorrência do evento. Para que as mesmas possam ser tomadas deve-se procurar reconhecer os fatores que se associam de forma significativa com o BPN e sobre eles atuar, de forma racional e decidida.

$\mathrm{Na}$ análise da relação entre BPN e idade materna pode-se verificar uma associaçāo significativa do fenômeno com gestação em adolescente, mormente as muito jovens. Mardones ${ }^{7}$, Barros e $\mathrm{col}^{1}{ }^{1}$ e Berezin e col. ${ }^{2}$ já haviam enaltecido esse fato. Parece clara, pois, a necessidade de se atuar firmemente no sentido de orientar adolescentes no que tange ao processo reprodutivo, tentando diminuir o numero de gestações nessa faixa etária, principalmente abaixo dos 17 anos.

Ficou evidente também, no presente estudo, a transcendência da assistência pré-natal, que deve atingir toda a população. Foi marcante a diferença na freqüência de BPN entre mulheres que tiveram ou não esse tipo de assistência médica. São dados que coincidem com os de Schuler e Klinger ${ }^{9}$ e com os de Berezin e col. ${ }^{2}$ Mas ficou claro, ainda, que não somente a cobertura assistencial importa. A extensão e periodicidade das consultas tem um peso específico próprio. Cai verticalmente o nascimento de crianças com BPN à medida que aumenta o número de consultas.

Em relação ao número de gestaçōes prévias não foi possível mostrar grandes diferenças entre os vários grupos, quando se relacionou ao BPN, ainda que a aplicação do teste estatístico tenha posto à mostra uma associação significativa. Mas, Berezin e col. ${ }^{2}$ e Miller ${ }^{8}$ não encontraram relação entre BPN e partos anteriores.

Os efeitos deletérios do hábito de fumar sobre o evento perinatal já foram mostrados por vários autores, entre os quais Lippi e col. ${ }^{5}$ e Lippi col. ${ }^{6}$ No estudo atual ficou mostrado que o hábito de fumar associa-se significativamente com a ocorrência do BPN. Mostrou-se, ademais, que essa associação é verdadeira, independente do número de cigarros fumados. Barros e col. ${ }^{1}$ já haviam verificado que as taxas de recém-natos de baixo peso eram duas vezes maiores entre as fumantes que entre as não-fumantes. Também Miller ${ }^{8}$ e Schuler e Klinger ${ }^{9}$ haviam mostrado a associação significativa entre esses fatos. $E$ de se supor, pois, que atitudes de mobilização contra o fumo tenha efeito positivo no sentido de atenuar o número de nascimentos de crianças com baixo peso.

Sem dúvida, a idade gestacional ao parto é um dos fatores mais freqüentemente associados ao BPN. Mardones ${ }^{7} \mathrm{já}$ havia assinalado que $61,9 \%$ dos recém-natos de baixo peso eram de pré-termo. No presente trabalho verificou-se, também, que quase metade $(46,3 \%)$ dos que nascem abaixo de 37 semanas têm BPN. Verificou-se ainda que nas faixas mais avançadas da gestação a freqüência decresce intensamente, e de modo estatisticamente significativo. Mas, ao se analisar a Tabela $7 \mathrm{em}$ outro sentido, pode-se verificar que $61,8 \%$ dos recém-nascidos de baixo peso desta população conta com idade gestacional ao parto inferior a 37 semanas. Envidar esforços no sentido de evitar partos de pré-termo, por meio de medidas bem sistematizadas, é fator de grande efeito, no intuito de se reduzir a freqüência de BPN.

\section{CONCLUSÖES}

A freqüência de BPN encontrada $(15,9 \%)$ é extremamente elevada. No entanto, compara-se com a de países latino-americanos, embora haja discrepância com os dados provenientes dos Estados Unidos e mesmo de um país do leste europeu, a Hungria. Considerando-se o risco da morbidade e da mortalidade dessas crianças torna-se claro que há necessidade de grande empenho dos perinatologistas no atendimento e principalmente na profilaxia do evento.

A gravidez na adolescência é um dos fatores fortemente associados ao BPN, principalmente em mulheres com menos de 17 anos. $O$ processo educacional em relação aos fenômenos da reprodução humana é recomendável junto a esse grupo etário.

A assistência pré-natal deve ser obrigatória, extensa e intensa, pois sua falta responsabiliza-se pelo incremento da freqüência do BPN. Os números decrescem na proporção inversa do aumento do número de consultas.

O número de gestações prévias é, dentre os fatores estudados, o de menor importância na gênese do BPN. 
O hábito de fumar, reconhecidamente deletério para o ganho de peso do feto intra-utero, associa-se significativamente com a freqüência do BPN. O consumo de cigarros é outra questão em relação a quais programas educacionais devidamente elaborados e executados podem surtir efeitos positivos.
A prematuridade é o fator mais conhecido e significativamente associado ao BPN. Programas de bloqueio do parto prematuro sāo úteis para minimizar o problema, embora essa medida médica deva surtir efeitos melhores se acompanhada de outras, de ordem sócio-econômica.

LIPPI, U.G. et al. [A study of some obstetric factors associated with low birth-weight]. Rev. Saúde públ, S. Paulo, 23: 382-7-, 1989.

ABSTRACT: An analysis was made of 18,804 of 19,446 consecutive births of the number analysed $15.93 \%$ presented low birth-rate. Significant statistical association was found in relation to maternal age, pre-natal care, previous pregnancies, smoking and gestational age at birth. Measures with a view to the attenuation of the problem are proposed, among them being: educational programs for teenagers on human reproduction, programs designed to create awareness of the harm done by smoking, amplification of antenatal assistance, medical programs for the limitation of premature labor, all of these and others, in association with programs of socio-economic support.

KEYWORDS: Infant, low birth weight. Prevalence. Risk factors.

\section{REFERENCIAS BIBLIOGRÁFICAS}

1. BARROS, F.C.; VICTORA, C.G.; GRANZOTO, J.A.; VAUGHAN, J.P.; LEMOS JR., A.V.Saúde perinatal em Pelotas, RS, Brasil: fatores sociais e biológicos. Rev. Saúde públ, S. Paulo, 18:301-12, 1984.

2. BEREZIN, A.; PACHI, P.R.; GUEDES, M.L.; ROCHA, E.E.A.; ARIE, E.; HERNANDES, F.L.M.; SILVA, M.M.; HANRA, A. Fatores maternos e fetais associados ao nascimento de prematuros com 1000 gramas ou menos. J. Pediat., 57:149-54, 1984.

3. HUTCHINS, V.; KESSEL, S.S.; PLACEK, PJ. Trends in matemal and infant health factors associated with low infant birth weight, United States, 1972 and 1980. Publ Hlth Rep., 99:162-72, 1984.

4. LIPPI, U.G.; SEGRE, C.A.M.; ANDRADE, A.S.; NASCIMENTO, RC.; REGEN, J.B. Coleta e análise de dados perinatais. Femina, 12:607-12, 1984.

5. LIPPI, U.G.; SEGRE, C.A.M.; ANDRADE, S.A.; COSTA, H.P.F.; MELLO, E. Fumo e gravidez. I - Influência sobre idade gestacional ao parto e peso ao nascer. Rev. poul. Pediat, 12:10-5, 1986.

6. LIPPI, U.G.; SEGRE, C.A.M.; ANDRADE, A.S.; COSTA, H.P.F.; MELLO, E. Fumo e gravidez. II - Influência sobre a vitalidade ao nascer, a morbilidade e a mortalidade conceptual. Rev. paul. Pediat, 12:16-9, 1986.

7. MARDONES, F. Algunos factores condicionantes del bajo peso de nascimiento. Rev. med. Chile, 108:839-52, 1980.

8. MILLER, H. A model for studying the pathogenesis and incidence of low-birthweight infants. Amer. J. Dis. Child, 137:323-, 1983.

9. SCHULER, D. \& KLINGER, A. Causes of low birthweight in Hungary. Acta Paediatr. Hung, 25:173-, 1984.

10. SCHWARCZ, R.; DIAZ, A.G.; FESCINA, R; ROSSELLO, J.L.D.; BELITZKY, R; MARTELL, M. Bajo peso al nacer y mortalidad perinatal en maternidades da América Latina. In: Organización Panamericana de la Salud. Salud maternoinfantil y atención primbria en las Américas. México, 1984. p.105-17 (Publicación científica, 461).

11. YOSHIDA-ANDO, P. \& MENDOZA-PÉREZ, A.M. Estudio sobre el recién nacido de peso subnormal. I Etiologia. Gac. med. MEx, 118:101-4, 1982.

Recebido para publicaçāo em 25/11/1988 Reapresentado em 12/7/1989 Aprovado para publicação em 13/7/1989 